\title{
On Solutions of Marginal Problem in Evidence Theory
}

\author{
Jiřina Vejnarová( $\left.{ }^{(}\right)(\mathbb{D}$ \\ Czech Academy of Sciences, Institute of Information Theory and Automation, \\ Pod Vodárenskou Věží 4, Prague, Czech Republic \\ vejnar@utia.cas.cz
}

\begin{abstract}
Recently introduced marginal problem - which addresses the question of whether or not a common extension exists for a given set of marginal basic assignments - in the framework of evidence theory is recalled. Sets of solutions are studied in more detail and it is shown, by a simple example, that their structure is much more complicated (i.e. the number of extreme vertices of the convex set of solutions is substantially greater) than that in an analogous problem in probabilistic framework. The concept of product extension of two basic assignments is generalized (via operator of composition) to a finite sequence of basic assignments. This makes possible not only to express the extension, if it exists, in a closed form, but also enables us to find the sufficient condition for the existence of an extension of evidential marginal problem. Presented approach is illustrated by a simple example.
\end{abstract}

Keywords: Marginal problem $\cdot$ Extension $\cdot$ Product extension

\section{Introduction}

The marginal problem is one of the most challenging problem types in probability theory. It addresses the question of whether or not a common extension exists for a given set of marginal distributions. The challenges lie not only in a wide range of the relevant theoretical problems (probably the most important among them is to find conditions for the existence of a solution to this problem), but also in its applicability to various problems of artificial intelligence [11], statistics [4] and computer tomography [6]. The importance of this problem type is emphasized by the fact that from 1990 a series of conferences with this topic takes place every three years. During the last two decades it has also been studied in other frameworks, for example, in possibility theory [9] and quantum mathematics [7].

In [10] we introduced an evidential marginal problem analogous to that encountered in the probabilistic framework. We demonstrated the similarities between these frameworks concerning necessary conditions and convexity of sets of solutions. We also introduced the concept of product extension of two marginal basic assignments.

The support of Grant No. GAČR 19-04579S is gratefully acknowledged.

(C) Springer Nature Switzerland AG 2020

M.-J. Lesot et al. (Eds.): IPMU 2020, CCIS 1238, pp. 382-393, 2020.

https://doi.org/10.1007/978-3-030-50143-3_29 
This paper is a natural continuation of the above-mentioned one. We study the convex sets of solutions in more details and reveal one substantial difference concerning the complexity of sets of all extensions between probabilistic and evidential frameworks. Nevertheless, the main attention is devoted to the generalization of product extension to finite sets of marginal basic assignments. This generalization is realized via operator of composition introduced in [5], which makes possible to express the product extension of a sequence of marginal basic assignments in an elegant way. This finally allows us to find sufficient condition for the existence of evidential marginal problem solution.

The paper is organised as follows: after a brief overview of necessary concepts and notation (Sect. 2) we start Sect. 3 by a motivation example, then we recall the evidential marginal problem (and what was done in [10]) and we also study convex sets of extensions. In Sect. 4 we deal with generalization of product extension to finite sets of basic assignments and find a sufficient condition for the existence of an extension. Finally, before the Conclusions we present a solution of the motivation example via perfect sequence model.

\section{Basic Concepts and Notation}

In this section we will, as briefly as possible, recall basic concepts from evidence theory [8] concerning sets and set functions.

\subsection{Set Projections and Extension}

For an index set $N=\{1,2, \ldots, n\}$, let $\left\{X_{i}\right\}_{i \in N}$ be a system of variables, each $X_{i}$ having its values in a finite set $\mathbf{X}_{i}$. In this paper we will deal with a multidimensional frame of discernment

$$
\mathbf{X}_{N}=\mathbf{X}_{1} \times \mathbf{X}_{2} \times \ldots \times \mathbf{X}_{n}
$$

and its subframes (for $K \subseteq N$ )

$$
\mathbf{X}_{K}=X_{i \in K} \mathbf{X}_{i}
$$

Throughout this paper, $X_{K}$ will denote a group of variables $\left\{X_{i}\right\}_{i \in K}$ when dealing with groups of variables on these subframes.

For $M \subset K \subseteq N$ and $A \subset \mathbf{X}_{K}$, we denote by $A^{\downarrow M}$ a projection of $A$ into $\mathbf{X}_{M}$ :

$$
A^{\downarrow M}=\left\{y \in \mathbf{X}_{M} \mid \exists x \in A: y=x^{\downarrow M}\right\},
$$

where, for $M=\left\{i_{1}, i_{2}, \ldots, i_{m}\right\}$,

$$
x^{\downarrow M}=\left(x_{i_{1}}, x_{i_{2}}, \ldots, x_{i_{m}}\right) \in \mathbf{X}_{M} .
$$

In addition to the projection, in this text we will also need its inverse operation that is usually called a cylindrical extension. The cylindrical extension of $A \subset \mathbf{X}_{K}$ to $\mathbf{X}_{L}$ $(K \subset L)$ is the set

$$
A^{\uparrow L}=\left\{x \in \mathbf{X}_{L}: x^{\downarrow K} \in A\right\}=A \times \mathbf{X}_{L \backslash K}
$$


A more complex instance is to make a common extension of two sets, which will be called a join [1]. By a join of two sets $A \subseteq \mathbf{X}_{K}$ and $B \subseteq \mathbf{X}_{L}(K, L \subseteq N)$, we will understand a set

$$
A \bowtie B=\left\{x \in \mathbf{X}_{K \cup L}: x^{\downarrow K} \in A \quad \& \quad x^{\downarrow L} \in B\right\} .
$$

Let us note that, for any $C \subseteq \mathbf{X}_{K \cup L}$, it naturally holds $C \subseteq C^{\downarrow K} \bowtie C^{\downarrow L}$, but generally $C \neq C^{\downarrow K} \bowtie C^{\downarrow L}$.

Let us also note that if $K$ and $L$ are disjoint, then the join of $A$ and $B$ is just their Cartesian product, $A \bowtie B=A \times B$, and if $K=L$ then $A \bowtie B=A \cap B$. If $K \cap L \neq \emptyset$ and $A^{\downarrow K \cap L} \cap B^{\downarrow K \cap L}=\emptyset$ then $A \bowtie B=\emptyset$ as well. Generally, $A \bowtie B=A^{\uparrow K \cup L} \cap B^{\uparrow K \cup L}$, i.e., a join of two sets is the intersection of their cylindrical extensions.

\subsection{Set Functions}

In evidence theory [8], two dual measures are used to model the uncertainty: belief and plausibility measures. Each of them can be defined with the help of another set function called a basic (probability or belief) assignment $m$ on $\mathbf{X}_{N}$, i.e.,

$$
m: \mathscr{P}\left(\mathbf{X}_{N}\right) \longrightarrow[0,1]
$$

where $\mathscr{P}\left(\mathbf{X}_{N}\right)$ is the power set of $\mathbf{X}_{N}$, and

$$
\sum_{A \subseteq \mathbf{X}_{N}} m(A)=1 .
$$

Furthermore, we assume that $m(\emptyset)=0 .{ }^{1}$ A set $A \in \mathscr{P}\left(\mathbf{X}_{N}\right)$ is a focal element if $m(A)>0$.

For a basic assignment $m$ on $\mathbf{X}_{K}$ and $M \subset K$, a marginal basic assignment of $m$ on $\mathbf{X}_{M}$ is defined (for each $A \subseteq \mathbf{X}_{M}$ ) by the equality

$$
m^{\downarrow M}(A)=\sum_{\substack{B \subseteq \mathbf{X}_{K} \\ B^{\downarrow M}=A}} m(B) .
$$

In this paper we will confine ourselves to basic assignments.

\section{Marginal Problem and Its Solutions}

In this section we first recall what we understand under evidential marginal problem, then we briefly discuss the necessary condition for the existence of a solution and finally study in more detail convex sets of its solutions.

\footnotetext{
${ }^{1}$ This assumption is not generally accepted, e.g., in [2] it is omitted.
} 


\subsection{Motivation Example-Poll Opinion}

Let us consider the following situation. We have obtained poll opinion contained in the left part of Table 1 expressing the dependence of preferences $P$ with values $A$ and $B$ on sex $S(M, F)$. Some voters are still undecided, we denote this fact by $\{A, B\}$.

From last census we can get data concerning education $E$ (primary $-P$, high school $-H$ and university $-U$ ) and place of residence $R$ (town $-T$ and countryside$C$ ) both of them with respect to sex. They are contained in the middle and right part of of Table 1, respectively. In both cases some values, denoted by $\{P, H, U\}$ and $\{T, C\}$ are missing.

Throughout this example we will express values of basic assignments in the percentage form in order to avoid to small numbers in the joint model.

Table 1. Motivation example: poll opinion and census.

\begin{tabular}{c|r}
\hline$S \& P$ & percentage \\
\hline$M, A$ & 19 \\
$M, B$ & 23 \\
$M,\{A, B\}$ & 7 \\
$F, A$ & 25 \\
$F, B$ & 16 \\
$F,\{A, B\}$ & 10 \\
\hline
\end{tabular}

\begin{tabular}{c|r}
\hline$S \& E$ & percentage \\
\hline$M, P$ & 24 \\
$M, H$ & 15 \\
$M, U$ & 7 \\
$M,\{P, H, U\}$ & 3 \\
$F, P$ & 18 \\
$F, H$ & 23 \\
$F, U$ & 8 \\
$F,\{P, H, U\}$ & 2 \\
\hline
\end{tabular}

\begin{tabular}{c|r}
\hline$S \& R$ & percentage \\
\hline$M, T$ & 22 \\
$M, C$ & 25 \\
$M,\{T, C\}$ & 2 \\
$F, T$ & 27 \\
$F, C$ & 23 \\
$F,\{T, C\}$ & 1 \\
\hline
\end{tabular}

Our aim is to get more detailed information about the voters by integrating information from all these tables together. Undecided voters and missing data suggest that this problem has to be modeled by evidence theory. In Sect. 5 one can find solution via method presented in this paper, more precisely in Sect. 4.

\subsection{Definition}

The evidential marginal problem was, analogous to probability theory, introduced in [10] as follows: Let us assume that $X_{i}, i \in N, 1 \leq|N|<\infty$ are finitely-valued variables, $\mathscr{K}$ is a system of nonempty subsets of $N$ and

$$
\mathscr{S}=\left\{m_{K}, K \in \mathscr{K}\right\}
$$

is a family of basic assignments, where each $m_{K}$ is a basic assignment on $\mathbf{X}_{K}$.

The problem we are interested in is the existence of an extension, i.e., a basic assignment $m$ on $\mathbf{X}$ whose marginals are basic assignments from $\mathscr{S}$; or, more generally, the set

$$
\mathscr{E}=\left\{m: m^{\downarrow K}=m_{K}, K \in \mathscr{K}\right\}
$$

is of interest. 
In the above-mentioned paper we proved that the necessary condition for the existence of an extension of basic assignments from $\mathscr{S}$ is their pairwise projectivity. Having two basic assignments $m_{1}$ and $m_{2}$ on $\mathbf{X}_{K}$ and $\mathbf{X}_{L}$, respectively $(K, L \subseteq N)$, we say that these assignments are projective if

$$
m_{1}^{\downarrow K \cap L}=m_{2}^{\downarrow K \cap L} .
$$

If set $\mathscr{S}$ consists of just two basic assignments this condition is also sufficient, but in more general case it need not be, as we showed in [10].

\subsection{Sets of Solutions}

In [10] we started to study also sets of all solutions of a marginal problem. We proved that, analogous to probabilistic framework, the set is convex. Nevertheless, as suggests the following simple example, resulting convex set is much more complicated.

Example 1. Consider, for $i=1,2$, two basic assignments $m_{i}$ on $\mathbf{X}_{i}=\left\{a_{i}, b_{i}\right\}$, specified in Table 2. Our task is to find basic assignments on $\mathbf{X}_{1} \times \mathbf{X}_{2}$ which are extreme points of the set $\mathscr{E}\left(m_{1}, m_{2}\right)$. We found the 23 extreme points ${ }^{2}$ summarized in Table 3 . Let us note, that any of basic assignments $m^{2-7}, m^{9-14}$ and $m^{16-21}$ stands for six basic assignments, where three focal elements are fixed (as suggested in the table) and the fourth one is one set $A$ from

$$
\begin{aligned}
\mathscr{A}= & \left\{\mathbf{X}_{1} \times \mathbf{X}_{2} \backslash\left\{\left(a_{1}, a_{2}\right)\right\}, \mathbf{X}_{1} \times \mathbf{X}_{2} \backslash\left\{\left(a_{1}, b_{2}\right)\right\}, \mathbf{X}_{1} \times \mathbf{X}_{2} \backslash\left\{\left(b_{1}, a_{2}\right)\right\},\right. \\
& \left.\mathbf{X}_{1} \times \mathbf{X}_{2} \backslash\left\{\left(b_{1}, b_{2}\right)\right\},\left\{\left(a_{1}, a_{2}\right),\left(b_{1}, b_{2}\right)\right\},\left\{\left(a_{1}, b_{2}\right),\left(b_{1}, a_{2}\right)\right\}\right\},
\end{aligned}
$$

with the values contained in the last column.

Table 2. Example 1: basic assignments $m_{1}$ and $m_{2}$.

\begin{tabular}{c|cccc}
\hline$A \subseteq \mathbf{X}_{1}$ & $m_{1}(A)$ & & $A \subseteq \mathbf{X}_{2}$ & $m_{2}(A)$ \\
\cline { 1 - 2 } \cline { 4 - 5 }$\left\{a_{1}\right\}$ & 0.2 & & $\left\{a_{2}\right\}$ & 0.6 \\
$\left\{b_{1}\right\}$ & 0.3 & & $\left\{b_{2}\right\}$ & 0 \\
$\mathbf{X}_{1}$ & 0.5 & & $\mathbf{X}_{2}$ & 0.4 \\
\hline
\end{tabular}

On the other hand, although they are numerous, they are quite "nice", as any of them has only four focal elements in contrary to the basic assignments from the interior of $\mathscr{E}$, any of which has twelve focal elements (from possible 15 in case of two binary variables). An example of these basic assignments is $m^{i}$, presented in last line of the table, a convex combination of $m^{1}, \ldots, m^{22}$, where, in contrary to $m^{1}, \ldots, m^{22}$ each set from $\mathscr{A}$ is a focal element with the same value (as indicated in the table).

From this point of view a deeper study of extreme points of solutions seems to be of importance. We intend to concentrate to this problem in the future research.

\footnotetext{
${ }^{2}$ Let us note that in analogous case of two binary variables in probabilistic framework the resulting convex set has two extreme points.
} 
Table 3. Example 1: extreme points of $\mathscr{E}\left(m_{1}, m_{2}\right)$.

\begin{tabular}{l|l|l|l|l|l|l|l}
\hline & $\left\{\left(a_{1}, a_{2}\right)\right\}$ & $\left\{\left(b_{1}, a_{2}\right)\right\}$ & $\left\{a_{1}\right\} \times \mathbf{X}_{2}$ & $\left\{b_{1}\right\} \times \mathbf{X}_{2}$ & $\mathbf{X}_{1} \times\left\{a_{2}\right\}$ & $\mathbf{X}_{1} \times \mathbf{X}_{2}$ & $A$ \\
\hline$m^{1}$ & 0.2 & 0.3 & 0 & 0 & 0.1 & 0.4 & 0 \\
$m^{2-7}$ & 0.2 & 0.3 & 0 & 0 & 0.1 & 0 & 0.4 \\
$m^{8}$ & 0.2 & 0 & 0 & 0.3 & 0.4 & 0.1 & 0 \\
$m^{9-14}$ & 0.2 & 0 & 0 & 0.3 & 0.4 & 0 & 0.1 \\
$m^{15}$ & 0 & 0.3 & 0.2 & 0 & 0.3 & 0.2 & 0 \\
$m^{16-21}$ & 0 & 0.3 & 0.2 & 0 & 0.3 & 0 & 0.2 \\
$m^{22}$ & 0 & 0.1 & 0.2 & 0.2 & 0.5 & 0 & 0 \\
$m^{23}$ & 0.1 & 0 & 0.1 & 0.3 & 0.5 & 0 & 0 \\
\hline$m^{i}$ & 0.1 & 0.205 & 0.1 & 0.095 & 0.295 & 0.07 & 0.0225 \\
\hline
\end{tabular}

\section{Product Extension of Sets of Marginals}

In this section we will first recall the concept of product extension [10] as well as the composition operator [5] of two basic assignments. We will show that the former is a special case of the latter. Then we use this relationship in order to generalize product extension to finite sets of basic assignments and finally to find a sufficient condition for the existence of an extension.

\subsection{Product Extensions}

Dempster's rule of combination [8] (and its various modifications as e.g. [3]) is the usual way to combine, in the framework of evidence theory, information from different sources. It is quite natural that several attempts were done to use it in order to merge information expressed by marginal basic assignments. Nevertheless none of them was able to keep both marginals even in case of projective basic assignments. We discussed this problem in more detail in [10].

Instead of the use of Dempster's rule we suggested to use product extension of marginal basic assignments defined as follows.

Definition 1. Let $m_{1}$ and $m_{2}$ be projective basic assignments on $\mathbf{X}_{K}$ and $\mathbf{X}_{L}(K, L \subseteq N)$, respectively. We will call basic assignment $m$ on $\mathbf{X}_{K \cup L}$ product extension of $m_{1}$ and $m_{2}$ if for any $A=A^{\downarrow K} \bowtie A^{\downarrow L}$

$$
m(A)=\frac{m_{1}^{\downarrow K}\left(A^{\downarrow K}\right) \cdot m_{2}^{\downarrow L}\left(A^{\downarrow L}\right)}{m_{1}^{\downarrow K \cap L}\left(A^{\downarrow K \cap L}\right)},
$$

whenever the right-hand side is defined, and $m(A)=0$ otherwise.

Let us note that the expression on the right-hand side of (4) is only seemingly asymmetric, as $m_{1}$ and $m_{2}$ are supposed to be projective. Therefore, it is irrelevant which marginal is used in the denominator.

From the following theorem proven in [10] one can easily see that product extension is superior to Dempster's rule as concerns keeping marginals. 
Theorem 1. Let $m_{1}$ and $m_{2}$ be two projective basic assignments on $\mathbf{X}_{K}$ and $\mathbf{X}_{L}(K, L \subseteq$ $N)$, respectively, and $m$ be their product extension. Then

$$
\begin{aligned}
& m^{\downarrow K}(B)=m_{1}(B), \\
& m^{\downarrow L}(C)=m_{2}(C)
\end{aligned}
$$

for any $B \in \mathbf{X}_{K}$ and $C \in \mathbf{X}_{L}$, respectively.

\subsection{Composition Operator}

Now, let us recall the concept of composition operator, introduced in [5].

Definition 2. For two arbitrary basic assignments $m_{1}$ on $\mathbf{X}_{K}$ and $m_{2}$ on $\mathbf{X}_{L}$ a composition $m_{1} \triangleright m_{2}$ is defined for all $A \subseteq \mathbf{X}_{K \cup L}$ by one of the following expressions:

[a] if $m_{2}^{\downarrow K \cap L}\left(A^{\downarrow K \cap L}\right)>0$ and $A=A^{\downarrow K} \bowtie A^{\downarrow L}$ then

$$
\left(m_{1} \triangleright m_{2}\right)(A)=\frac{m_{1}\left(A^{\downarrow K}\right) \cdot m_{2}\left(A^{\downarrow L}\right)}{m_{2}^{\downarrow K \cap L}\left(A^{\downarrow K \cap L}\right)} ;
$$

[b] if $m_{2}^{\downarrow K \cap L}\left(A^{\downarrow K \cap L}\right)=0$ and $A=A^{\downarrow K} \times \mathbf{X}_{L \backslash K}$ then

$$
\left(m_{1} \triangleright m_{2}\right)(A)=m_{1}\left(A^{\downarrow K}\right) ;
$$

[c] in all other cases

$$
\left(m_{1} \triangleright m_{2}\right)(A)=0 .
$$

The purpose of the composition operator is to integrate information from two sources expressed by basic assignments $m_{1}$ and $m_{2}$. The resulting basic assignment $m_{1} \triangleright m_{2}$ has to keep all the information contained in the first basic assignment, and as much as possible from the second one.

The definition completely fulfills this requirement. The only focal elements are those obtained via $[\mathbf{a}]$ or $[\mathbf{b}]$. Both of them keep the first marginal, i.e. all the information contained in $m_{1}$.

In case $[\mathbf{a}]$ "as much as possible from the second basic assignment" is obtained via multiplication by $m_{2}$ (divided by its marginal). If $m_{1}$ and $m_{2}$ are projective, then also all the information from $m_{2}$ is kept ${ }^{3}$ (cf. Theorem 1 and Lemma 1).

In case [b] no information about $A^{\downarrow K \cap L}$ is available, so $m_{1} \triangleright m_{2}$ is the least specific basic assignment with marginal $m_{1}$ - its vacuous extension.

The following lemma reveals, that product extension of two basic assignments is a special case of composition of two basic assignments.

Lemma 1. Let $m_{1}$ and $m_{2}$ be projective basic assignments. Then $\left(m_{1} \triangleright m_{2}\right)(A)$ is equal to their product extension defined by (4).

Proof. First, let us note that when $m_{1}$ and $m_{2}$ are projective, case [b] is not applied. Therefore, $\left(m_{1} \triangleright m_{2}\right)(A)$ is computed via $[\mathbf{a}]$, which is equal to the right hand side of (4) (due to projectivity of $m_{1}$ and $\left.m_{2}\right)$, whenever it is defined. Otherwise, by [c] $\left(m_{1} \triangleright\right.$ $\left.m_{2}\right)(A)=0$.

\footnotetext{
${ }^{3}$ Let us note that if $K$ and $L$ are disjoint, then $m_{1}$ and $m_{2}$ are trivially projective.
} 


\subsection{Iterative Application of Composition Operator}

Composing of $m_{1}, m_{2}, \ldots, m_{n}$ defined on $\mathbf{X}_{K_{1}}, \ldots, \mathbf{X}_{K_{n}}$, respectively, together by multiple application of the operator of composition, one gets multidimensional basic assignments on $\mathbf{X}_{K_{1} \cup K_{2} \cup \ldots \cup K_{n}}$. However, since the operator of composition is neither commutative nor associative (cf. [5]), we have to specify what "composing them together" means.

To avoid using too many brackets let us make the following convention. Whenever we put down the expression $m_{1} \triangleright m_{2} \triangleright \ldots \triangleright m_{n}$ we will understand that the operator of composition is performed successively from left to right: ${ }^{4}$

$$
m_{1} \triangleright m_{2} \triangleright \ldots \triangleright m_{n}=\left(\ldots\left(\left(m_{1} \triangleright m_{2}\right) \triangleright m_{3}\right) \triangleright \ldots\right) \triangleright m_{n} .
$$

Therefore, it is obvious that ordering of basic assignments in question is substantial for the properties of the resulting model. Let us demonstrate it by the following simple example.

Example 2. Consider three variables $X_{1}, X_{2}$ and $X_{3}$ with values in $\mathbf{X}_{1}, \mathbf{X}_{2}$ and $\mathbf{X}_{3}$, respectively, $\mathbf{X}_{i}=\left\{a_{i}, b_{i}\right\}, i=1,2,3$. Let $m_{1}, m_{2}$ and $m_{3}$ be defined as shown in Table 4 .

Table 4. Example 2: basic assignments $m_{1}, m_{2}$ and $m_{3}$.

\begin{tabular}{c|c}
\hline$A \subseteq \mathbf{X}_{1} \times \mathbf{X}_{2}$ & $m_{1}(A)$ \\
\hline$\left\{\left(a_{1}, a_{2}\right)\right\}$ & 0.5 \\
$\mathbf{X}_{1} \times \mathbf{X}_{2}$ & 0.5 \\
\hline
\end{tabular}

\begin{tabular}{c|c}
\hline$A \subseteq \mathbf{X}_{2} \times \mathbf{X}_{3}$ & $m_{2}(A)$ \\
\hline$\left\{\left(a_{2}, a_{3}\right)\right\}$ & 0.5 \\
$\mathbf{X}_{2} \times \mathbf{X}_{3}$ & 0.5 \\
\hline
\end{tabular}

\begin{tabular}{c|c}
\hline$A \subseteq \mathbf{X}_{1} \times \mathbf{X}_{3}$ & $m_{3}(A)$ \\
\hline$\left\{a_{1}\right\} \times \mathbf{X}_{3}$ & 0.5 \\
$\mathbf{X}_{1} \times\left\{a_{3}\right\}$ & 0.5 \\
\hline
\end{tabular}

These basic assignments are pairwise projective (any one-dimensional marginal has just two focal elements, namely $\left\{a_{i}\right\}$ and $\mathbf{X}_{i}$ ), but common extension does not exist (as we already showed in [10]). One can judge that application of the operator of composition to different orderings of these three basic assignments will lead to different joint basic assignments on $\mathbf{X}_{1} \times \mathbf{X}_{2} \times \mathbf{X}_{3}$. And it is the case. Each of these composed basic assignments has again only two focal elements, namely

$$
\left\{\left(a_{1}, a_{2}, a_{3}\right)\right\}, \mathbf{X}_{1} \times \mathbf{X}_{2} \times \mathbf{X}_{3}
$$

for $m_{1}, m_{2}, m_{3}$ and $m_{2}, m_{1}, m_{3}$,

$$
\left\{\left(a_{1}, a_{2}\right)\right\} \times \mathbf{X}_{3}, \mathbf{X}_{1} \times \mathbf{X}_{2} \times\left\{a_{3}\right\}
$$

for $m_{1}, m_{3}, m_{2}$ and $m_{3}, m_{1}, m_{2}$ and, finally,

$$
\mathbf{X}_{1} \times\left\{\left(a_{2}, a_{3}\right)\right\},\left\{a_{1}\right\} \times \mathbf{X}_{2} \times \mathbf{X}_{3}
$$

for $m_{2}, m_{3}, m_{1}$ and $m_{3}, m_{2}, m_{1}$.

In the next section we will deal with special kind of sequences of basic assignments.

\footnotetext{
${ }^{4}$ Naturally, if we want to change the ordering in which the operators are to be performed we will do so with the help of brackets.
} 


\subsection{Perfect Sequences and Sufficient Condition}

When representing knowledge in a specific area of interest, a special role is played by the so-called perfect sequences, i.e., sequences $m_{1}, m_{2}, \ldots, m_{n}$, for which

$$
\begin{aligned}
m_{1} \triangleright m_{2} & =m_{2} \triangleright m_{1}, \\
m_{1} \triangleright m_{2} \triangleright m_{3} & =m_{3} \triangleright\left(m_{1} \triangleright m_{2}\right), \\
& \vdots \\
m_{1} \triangleright m_{2} \triangleright \ldots \triangleright m_{n} & =m_{n} \triangleright\left(m_{1} \triangleright \ldots \triangleright m_{n-1}\right) .
\end{aligned}
$$

The following theorem proven in [5] explains why perfect sequences are useful for marginal problem solution.

Theorem 2. A sequence $m_{1}, m_{2}, \ldots, m_{n}$ is perfect if and only if all $m_{1}, m_{2}, \ldots, m_{n}$ are marginal basic assignments of the multidimensional basic assignment $m_{1} \triangleright m_{2} \triangleright \ldots \triangleright m_{n}$ :

$$
\left(m_{1} \triangleright m_{2} \triangleright \ldots \triangleright m_{n}\right)^{\downarrow K_{i}}=m_{i},
$$

for all $i=1, \ldots, n$.

Let us note that this theorem expresses necessary and sufficient condition for the existence of an extension of basic assignments from $\mathscr{S}$, however, this condition is hardly verifiable as it is obvious from the definition of the perfect sequence. Nevertheless, we can formulate a sufficient condition expressed by Theorem 3. Before doing it, let us recall the well-known running intersection property and a lemma (proven in [5]) necessary to prove Theorem 3.

We say that $K_{1}, K_{2}, \ldots, K_{n}$ meets the running intersection property iff

$$
\forall i=2,3, \ldots, n \exists j(1 \leq j<i) \quad \text { such that } K_{i} \cap\left(K_{1} \cup \ldots \cup K_{i-1}\right) \subseteq K_{j} \text {. }
$$

Lemma 2. A sequence $m_{1}, m_{2}, \ldots, m_{n}$ is perfect iff the pairs of basic assignments $m_{i}$ and $\left(m_{1} \triangleright \ldots \triangleright m_{i-1}\right)$ are projective, i.e. if

$$
m_{i}^{\left\lfloor K_{i} \cap\left(K_{1} \cup \ldots \cup K_{i-1}\right)\right.}=\left(m_{1} \triangleright \ldots \triangleright m_{i-1}\right)^{\downarrow K_{i} \cap\left(K_{1} \cup \ldots \cup K_{i-1}\right)},
$$

for all $i=2,3, \ldots, n$.

Theorem 3. Let $\mathscr{S}$ be a system of pairwise projective basic assignments on $\mathbf{X}_{K}, K \in$ $\mathscr{K}$, and let $\mathscr{K}$ be ordered in such a way that $K_{1}, K_{2}, \ldots, K_{n}$ meets running intersection property. Then $\mathscr{E} \neq \emptyset$.

Proof. We have to prove that the sequence of basic assignments $m_{1}, m_{2}, \ldots, m_{n}$ on $\mathbf{X}_{K_{1}}, \mathbf{X}_{K_{2}}, \ldots, \mathbf{X}_{K_{n}}$, respectively, is perfect. Then, according to Theorem $2\left(m_{1} \triangleright m_{2} \triangleright\right.$ $\left.\ldots \triangleright m_{n}\right) \in \mathscr{E}$.

Due to Lemma 2 it is enough to show that for each $i=2, \ldots, n$ basic assignment $m_{i}$ and the composed assignment $m_{1} \triangleright \ldots \triangleright m_{i-1}$ are projective. Let us prove it by induction.

For $i=2$ the required projectivity is guaranteed by the fact that we assume pairwise projectivity of all $m_{1}, \ldots, m_{n}$. So we have to prove it for general $i>2$ under the 
assumption that the assertion holds for $i-1$, which means (due to Theorem 2) that all $m_{1}, m_{2}, \ldots, m_{i-1}$ are marginal to $m_{1} \triangleright \ldots \triangleright m_{i-1}$. Since we assume that $K_{1}, \ldots, K_{n}$ meets the running intersection property, there exists $j \in\{1,2, \ldots i-1\}$ such that $K_{i} \cap\left(K_{1} \cup\right.$ $\left.\ldots \cup K_{i-1}\right) \subseteq K_{j}$. Therefore $\left(m_{1} \triangleright \ldots \triangleright m_{i-1}\right)^{\downarrow K_{i} \cap\left(K_{1} \cup \ldots \cup K_{i-1}\right)}$ and $m_{j}^{\downarrow K_{i} \cap\left(K_{1} \cup \ldots \cup K_{i-1}\right)}$ are the same marginals of $m_{1} \triangleright \ldots \triangleright m_{i-1}$ and therefore they have to equal to each other:

$$
\left(m_{1} \triangleright \ldots \triangleright m_{i-1}\right)^{\downarrow K_{i} \cap\left(K_{1} \cup \ldots \cup K_{i-1}\right)}=m_{j}^{\downarrow K_{i} \cap\left(K_{1} \cup \ldots \cup K_{i-1}\right)} .
$$

However we assume that $m_{i}$ and $m_{j}$ are projective and therefore also

$$
\left(m_{1} \triangleright \ldots \triangleright m_{i-1}\right)^{\downarrow K_{i} \cap\left(K_{1} \cup \ldots \cup K_{i-1}\right)}=m_{i}^{\downarrow K_{i} \cap\left(K_{1} \cup \ldots \cup K_{i-1}\right)} .
$$

Nevertheless, there exist a big "grey zone" of problems satisfying necessary condition but not the sufficient one. In this case the answer can be negative (as in Example 2) or positive (as in the following example).

Example 3. Consider again three variables $X_{1}, X_{2}$ and $X_{3}$ with values in $\mathbf{X}_{1}, \mathbf{X}_{2}$ and $\mathbf{X}_{3}$, respectively, $\mathbf{X}_{i}=\left\{a_{i}, b_{i}\right\}, i=1,2,3$. Let $m_{1}, m_{2}$ and $m_{3}^{\prime}$ be defined as shown in Table 5 . The only difference with Example 2 consists in using $m_{3}^{\prime}$ instead of $m_{3}$.

Table 5. Example 3: basic assignments $m_{1}, m_{2}$ and $m_{3}^{\prime}$.

\begin{tabular}{c|c}
\hline$A \subseteq \mathbf{X}_{1} \times \mathbf{X}_{2}$ & $m_{1}(A)$ \\
\hline$\left\{\left(a_{1}, a_{2}\right)\right\}$ & 0.5 \\
$\mathbf{X}_{1} \times \mathbf{X}_{2}$ & 0.5 \\
\hline
\end{tabular}

\begin{tabular}{c|c}
\hline$A \subseteq \mathbf{X}_{2} \times \mathbf{X}_{3}$ & $m_{2}(A)$ \\
\hline$\left\{\left(a_{2}, a_{3}\right)\right\}$ & 0.5 \\
$\mathbf{X}_{2} \times \mathbf{X}_{2}$ & 0.5 \\
\hline
\end{tabular}

\begin{tabular}{c|c}
\hline$A \subseteq \mathbf{X}_{1} \times \mathbf{X}_{3}$ & $m_{3}^{\prime}(A)$ \\
\hline$\left\{\left(a_{1}, a_{3}\right)\right\}$ & 0.5 \\
$\mathbf{X}_{1} \times \mathbf{X}_{3}$ & 0.5 \\
\hline
\end{tabular}

These basic assignments are again pairwise projective and the running intersection property for $\{1,2\},\{2,3\}$ and $\{1,3\}$ (for any ordering of these sets) does not again hold. However, in this case a common extensions exist; product extension can be found in Table 6.

Table 6. Example 3: product extension of $m_{1}, m_{2}$ and $m_{3}^{\prime}$.

\begin{tabular}{l|l}
\hline$A \subseteq \mathbf{X}_{1} \times \mathbf{X}_{2} \times \mathbf{X}_{3}$ & $m(A)$ \\
\hline$\left\{\left(a_{1}, a_{2}, a_{3}\right)\right\}$ & 0.5 \\
$\mathbf{X}_{1} \times \mathbf{X}_{2} \times \mathbf{X}_{3}$ & 0.5 \\
\hline
\end{tabular}

The difference between Examples 2 and 3 consists in the fact that $m_{1}, m_{2}$ and $m_{3}^{\prime}$ is perfect (although running intersection property is no satisfied) in contrary to $m_{1}, m_{2}$ and $m_{3}$.

From this example one can see, that perfectness does not depend only on relations among different subframes (or their index sets), on which low-dimensional basic assignments are defined, but also of the individual structure of any of them. 


\section{Poll Opinion-Solution}

It can be easily seen from Table 1 that $m_{1}, m_{2}$ and $m_{3}$ are pairwise projective, as

$$
m_{1}(M)=m_{2}(M)=m_{3}(M)=0.49
$$

and

$$
m_{1}(F)=m_{2}(F)=m_{3}(F)=0.51 .
$$

Since the sequence $\{S, O\},\{S, E\},\{S, R\}$, satisfies RIP, $m_{1}, m_{2}$ and $m_{3}$ form a perfect sequence. Application of composition operator to $m_{1}$ and $m_{2}$ and then to $m_{1} \triangleright m_{2}$ and $m_{3}$ gives rise to the joint basic assignment contained in Table 7 .

\begin{tabular}{|c|c|c|c|c|c|c|c|}
\hline & \multicolumn{3}{|c|}{ Male } & \multicolumn{3}{|c|}{ Female } \\
\hline & & $T$ & $C$ & $\{C, T\}$ & $T$ & $C$ & $\{C, T\}$ \\
\hline \multirow[t]{4}{*}{$P=A$} & $E=P$ & 4.19 & 4.75 & 0.38 & 4.67 & 3.98 & 0.17 \\
\hline & $E=H$ & 2.61 & 2.97 & 0.24 & 5.99 & 5.1 & 0.22 \\
\hline & $E=A$ & 1.21 & 1.38 & 0.11 & 2.07 & 1.76 & 0.08 \\
\hline & $E=\{P, H, U\}$ & 0.54 & 0.61 & 0.05 & 0.53 & 0.45 & 0.02 \\
\hline \multirow[t]{4}{*}{$P=B$} & $E=P$ & 5.08 & 5.78 & 0.46 & 2.96 & 2.53 & 0.11 \\
\hline & $E=H$ & 3.15 & 3.58 & 0.29 & 3.82 & 3.26 & 0.14 \\
\hline & $E=A$ & 1.49 & 1.69 & 0.13 & 1.33 & 1.13 & 0.05 \\
\hline & $E=\{P, H, U\}$ & 0.63 & 0.71 & 0.06 & 0.32 & 0.27 & 0.01 \\
\hline \multirow[t]{4}{*}{$P=\{A, B\}$} & $E=P$ & 1.54 & 1.74 & 0.14 & 1.86 & 1.57 & 0.07 \\
\hline & $E=H$ & 0.94 & 1.07 & 0.09 & 2.39 & 2.04 & 0.09 \\
\hline & $E=A$ & 0.45 & 0.51 & 0.04 & 0.85 & 0.72 & 0.03 \\
\hline & $E=\{P, H, U\}$ & 0.18 & 0.2 & 0.02 & 0.21 & 0.18 & 0.01 \\
\hline
\end{tabular}

Table 7. Poll opinion-solution

This table contains all focal elements of the basic assignment $m_{1} \triangleright m_{2} \triangleright m_{3}$, i.e only 72 from possible almost 17 million. Therefore it is evident, that despite the fact that evidential models are super-exponentially complex, compositional models belong (among them) to those with lower complexity. However, complexity of the resulting model strongly depends on the complexity of input basic assignments as well as on their number.

From this table one can obtain by simple marginalization marginal tables of the relationship between some variables of interest - e.g. preference and education.

\section{Conclusions}

We have recalled an evidential marginal problem introduced in [10] in a way analogous to a probability setting, where marginal probabilities are substituted by marginal basic assignments. 
We studied the structure of the sets of extensions of the problem in more detail and realized that it is much more complicated than that in analogous probabilistic case. This will be topic of our future research.

We also generalized concept of product extension to a finite set of basic assignments using the operator of composition introduced in [5]. The result of this effort is not only the closed form of an extension of a finite set of basic assignments (if it exists), but also the sufficient condition for the existence of such an extension. The obtained results are illustrated by a simple practical example.

\section{References}

1. Beeri, C., Fagin, R., Maier, D., Yannakakis, M.: On the desirability of acyclic database schemes. J. Assoc. Comput. Mach. 30(3), 479-513 (1983)

2. Yaghlane, B.B., Smets, P., Mellouli, K.: Belief functions independence: I. The marginal case. Int. J. Approximate Reasoning 29(1), 47-70 (2002)

3. Yaghlane, B., Smets, P., Mellouli, K.: Belief functions independence: II. The conditional case. Int. J. Approximate Reasoning 31(1-2), 31-75 (2002)

4. Janžura, M.: Marginal problem, statistical estimation, and Möbius formula. Kybernetika 43(5), 619-631 (2007)

5. Jiroušek, R., Vejnarová, J.: Compositional models and conditional independence in evidence theory. Int. J. Approximate Reasoning 52, 316-334 (2011)

6. Pougaza, D.-B., Mohammad-Djafaria, A., Berchera, J.-F.: Link between copula and tomography. Pattern Recogn. Lett. 31(14), 2258-2264 (2010)

7. Schilling, C.: The quantum marginal problem. In: Proceedings of the Conference QMath 12, Berlin (2013)

8. Shafer, G.: A Mathematical Theory of Evidence. Princeton University Press, Princeton (1976)

9. Vejnarová, J.: On possibilistic marginal problem. Kybernetika 43(5), 657-674 (2007)

10. Vejnarová, J.: On marginal problem in evidence theory. In: Cuzzolin, F. (ed.) Belief Functions: Theory and Applications, pp. 331-338 (2014)

11. Vomlel, J.: Integrating inconsistent data in a probabilistic model. J. Appl. Non-Class. Logics 14(3), 367-386 (2004) 